\title{
Iris Autonomic Function in Acute Glaucoma
}

\author{
D. J. BRAZIER
}

London

\begin{abstract}
Summary
Iris autonomic function was studied by binocular infrared pupillometry in 12 patients with acute primary angle closure glaucoma (APACG) and 28 control subjects. Light reflex amplitude and resting darkness pupil diameter were reduced and miotic response to pilocarpine $0.125 \%$ drops increased in APACG when compared to controls with anterior chamber depths (ACD) greater than $2.5 \mathrm{~mm}$ but not when compared to controls with ACD of $2.5 \mathrm{~mm}$ or less. These findings suggest relative autonomic dysfunction, predominantly parasympathetic, in the anterior segment of the eye in APACG.

Correlation between results of pupil tests and ACD suggested reduced autonomic function in eyes with shallow anterior chambers. Iris autonomic dysfunction in patients with APACG appears to be a reflection of shallow anterior chambers rather than a specific feature of the condition.
\end{abstract}

Whilst some factors predisposing to acute primary angle closure glaucoma (APACG), such as shallow anterior chambers ${ }^{1}$ and physiological pupil block, ${ }^{2}$ are well recognised , the chain of events determining the onset of most attacks remains uncertain. In some cases angle closure follows application of mydriatics, miotics ${ }^{3,4}$, or both, 5 acting by mechanisms including alteration of pupil size and physiological pupil block, change in anterior chamber depth and crowding of the drainage angle with iris tissue. ${ }^{2}$ Often, however, no precipitating factor can be identified.

In 1985 Clark and Mapstone ${ }^{6}$ suggested that abnormal ocular autonomic function might predispose certain individuals to APACG by modification of physiological pupil block. This theory was supported by the identification of a high incidence of abnormal cardiovascular autonomic function in patients with closed angle glaucoma and was consistent with previous work ${ }^{7}$ suggesting that the event preceding APACG was an alteration of autonomic activity in the eye. In order to clarify the type and degree of any such abnormality of ocular autonomic function, a pupillographic study of patients with APACG was undertaken at St Thomas' Hospital; the findings of this study are reported here.

\section{Subjects and Methods}

When informed consent had been obtained, twelve subjects with APACG and 28 healthy control subjects of both sexes joined the study which was approved by the ethical committee of West Lambeth Health District. APACG subjects were included if they had unilateral symptoms, a normal fellow eye and were able to cooperate with the measurements. Control subjects were recruited from general ophthalmic clinics when eye examination had excluded any disorder influencing pupil func- 
tion. Diabetics and subjects taking drugs or having medical disorders altering autonomic function were excluded. As the risk of APACG is greater in subjects with shallow anterior chambers, ${ }^{1,8}$ control subjects were divided into a group with shallow anterior chambers (SAC controls, anterior chamber depth $2.5 \mathrm{~mm}$ or less) and a group with deep anterior chambers (DAC controls, anterior chamber depth $>2.5 \mathrm{~mm}$ ). Anterior chamber depth was measured with the Haag-Streit meter. Subject characteristics are summarised in Table I.

Pupillary measurements were made with a Whittaker 1800 Series binocular infrared television pupillometer. The fellow eye was tested in all APACG subjects, one randomly chosen eye in controls. Light reflexes were elicited with light from tungsten arc lamps as previously described. ${ }^{9}$ After 30 minutes dark adaptation with red goggles, resting pupil diameter was measured in darkness. Visual perception threshold was determined in the test eye by a forced choice method. ${ }^{9}$ To determine the pupil response to a light stimulus six $\log$ units greater than perception threshold, ${ }^{10}$ five $0.5 \mathrm{sec}$ flashes were given at $8 \mathrm{sec}$ intervals and pupil diameter recorded continuously on a paper trace. Light reflex amplitude and diameter at the foot of the reflex were obtained by averaging the last four reflexes.

Stimulus light intensity was increased to 8 log units above threshold and three reflexes recorded with one sec stimuli $15 \mathrm{sec}$ apart. Time to redilate $3 / 4$ of the reflex amplitude after the stimulus ( $t 3 / 4)$ was averaged from the last two reflexes. $t 3 / 4$ is an assessment of iris sympathetic function based on the observations of Lowenstein and Loewenfeld. ${ }^{11}$

These measurements were made in all subjects on one occasion and on two separate occasions in seven APACG subjects (the day after presentation and some weeks later when all treatment had been stopped after laser iridotomy).

Drug studies of pupil function were performed in 8/12 APACG subjects, 7/8 SAC controls and 14/19 DAC controls with a different drug on each of three occasions separated by at least seven days. Three drug solutions were used; pilocarpine $0.125 \%$ to test for iris cholinoceptor supersensitivity, ${ }^{12}$ phenylephrine $2 \%$ to test for iris alpha adrenoceptor supersensitivity and hydroxyamphetamine $0.5 \%$, which causes release of noradrenaline from sympathetic nerve terminals, to assess the integrity of the postganglionic sympathetic neurone. Pilocarpine $0.125 \%$ and phenylephrine $2 \%$ were made by dilution of non-preserved solutions (Minims, Smith and Nephew). Hydroxyamphetamine $0.5 \%$ was made from powder Drug solutions were freshly prepared each day in $0.15 \mathrm{M}$ phosphate buffer and warmed to 34 degrees centigrade before instillation with a disposable pipette.

Procedure at each visit was the same. Pupil diameters were measured in both eyes with the pupillometer in the dark (pilocarpine) or standardised room lighting (phenylephrine and hydroxyamphetamine) prior to and 30,45 and 60 minutes after instillation of two drops, separated by thirty seconds, to the test eye The drug effect was recorded as the maximum anisocoria after treatment and took into account any physiological anisocoria at the control measurement.

As glaucoma and diabetes mellitus are known to be associated ${ }^{13-15}$ and pupil dysfunction a recognised feature of diabetic autonomic neuropathy, ${ }^{16,17}$ all subjects underwent oral glucose tolerance testing according to WHO guidelines. ${ }^{18}$ Each subject was classified as one of the following: normal (fasting glucose and $2 \mathrm{hr}$ post load $<6.7 \mathrm{mmol} / \mathrm{L}$ ), impaired glucose tolerance (fasting glucose

Table I. Subject characteristics

\begin{tabular}{lccc}
\hline & Number & $\begin{array}{c}\text { Age } \\
(\text { yrs, mean } \pm S D)\end{array}$ & $\begin{array}{c}\text { Anterior chamber depth } \\
\text { (mm, mean } \pm S D)\end{array}$ \\
\hline APACG & 12 & $71.0 \pm 10.9$ & $1.8 \pm 0.19$ \\
SAC control & 9 & $63.4 \pm 11.5$ & $2.3 \pm 0.20$ \\
DAC control & 19 & $65.0 \pm 11.4$ & $3.0 \pm 0.34$ \\
\hline
\end{tabular}


$<6.7 \mathrm{mmol} / \mathrm{L}, 2 \mathrm{hr}$ post load $6.7-10 \mathrm{mmol} . \mathrm{L}$ ) or diabetes mellitus (fasting glucose $>6.7$ $\mathrm{mmol} / \mathrm{L}$ and/or $2 \mathrm{hr}$ post load $>10 \mathrm{mmol} / \mathrm{L}$ ).

\section{Statistical Methods}

Results were compared between groups by unpaired Student's t tests. Sequential linear regression analysis was used to identify significant correlations between results of pupil tests and other variables in the data. In order to investigate the effect of significant correlations further, the regression equation was used in each case to produce expected values for all data points. Expected (E) values were then subtracted from observed $(\mathrm{O})$ and the residuals (the positive or negative value by which any data point varied from its expected value) compared between groups by unpaired $\mathrm{t}$ tests. For seven APACG subjects undergoing repeated measurements, results before and after laser iridotomy were compared by paired t tests. Results of glucose tolerance tests were compared between groups by chisquared.

\section{Results}

Comparison of results for resting darkness pupil diameter, light reflex amplitude and pilocarpine $0.125 \%$ miosis between groups showed significant differences between APACG subjects and DAC controls but no difference between APACG subjects and SAC controls (Table II). There was no difference between groups for $\mathrm{t} 3 / 4$, phenylephrine mydriasis and hydroxyamphetamine mydriasis.

Resting darkness pupil diameter showed a significant correlation with ACD $(r=0.35$ $\mathrm{p}>0.05$ ) (Fig. 1) but not age. When corrected for ACD using observed and expected values, the difference in resting darkness pupil diameter between APACG and DAC control groups became non-significant (Table III).

Light reflex amplitude correlated significantly with ACD ( $\mathrm{r}=0.40 \mathrm{p}>0.05)$ (Fig. 2) and diameter at foot of reflex ( $r=0.41$ $\mathrm{p}>0.01)$. When corrected for ACD, light reflex amplitude was not significantly different between groups (Table III). When corrected for diameter at the foot of the reflex, difference between APACG and DAC control subjects was enhanced $(\mathrm{p}>0.005)$ (Table III).

Pilocarpine $0.125 \%$ miosis correlated with both ACD $(r=-0.42 \mathrm{p}<0.05)$ (Fig. 3) and age $(r=0.53 p<0.01)$. When the age difference between groups was corrected by use of observed and expected values, the significant difference between APACG and DAC control subjects was unchanged (Table III). Correction for ACD made the difference between these groups non-significant (Table III).

Results for resting darkness pupil diameter, light reflex amplitude and $\mathrm{t} 3 / 4$ showed no significant difference between occasions when pre and post iridotomy measurements were compared.

Glucose tolerance testing showed no significant difference between the three groups. No subject was found to be diabetic. 2/12 APACG and 2/19 DAC controls showed impaired glucose tolerance, all other subjects being normal.

\section{Discussion}

Light reflex amplitude may be regarded as predominantly a measure of parasympathetic function of the iris. ${ }^{19}$ The magnitude of the miotic response to dilute pilocarpine reflects the degree of cholinoceptor sensitivity of the

Table II. Results of pupil tests (mean \pm SEM)

\begin{tabular}{lccc}
\hline & APACG & SAC Control & DAC Control \\
\hline Light reflex amplitude $(\mathrm{mm})$ & $0.89 \pm 0.11$ & $\begin{array}{c}1.04 \pm 0.14 \\
(\mathrm{NS})\end{array}$ & $\begin{array}{c}1.31 \pm 0.095 \\
(\mathrm{p}<0.01)\end{array}$ \\
Pilocarpine 0.125\% miosis $(\mathrm{mm})$ & $2.64 \pm 0.16$ & $\begin{array}{c}2.49 \pm 0.42 \\
(\mathrm{NS})\end{array}$ & $\begin{array}{r}1.91 \pm 0.22 \\
(\mathrm{p}<0.05)\end{array}$ \\
Resting darkness pupil diameter $(\mathrm{mm})$ & $4.99 \pm 0.28$ & $\begin{array}{c}5.69 \pm 0.36 \\
(\mathrm{NS})\end{array}$ & $\begin{array}{r}5.69 \pm 0.15 \\
(\mathrm{p}<0.05)\end{array}$ \\
\hline
\end{tabular}

Brackets indicate no significant difference (NS) or p value for unpaired t test between APACG and SAC control (shown in SAC control column) or APACG and DAC control (show in DAC control column). 


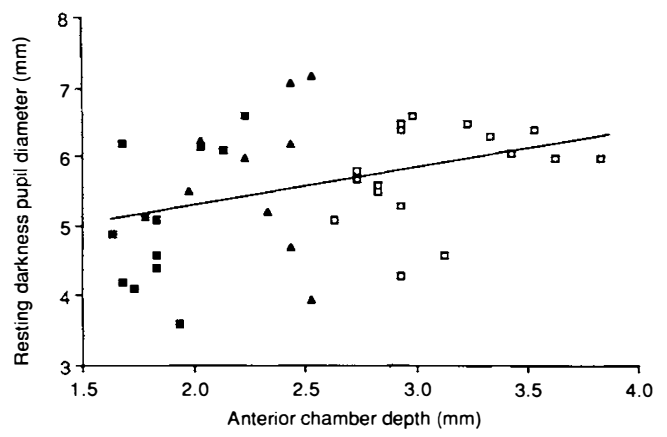

Fig. 1. Resting darkness pupil diameter for $A P A C G$ (ם), SAC controls ( $\mathbf{\Delta})$ and DAC controls ( $\square)$ correlated significantly with anterior chamber depth $(r=0.35$ $p<0.05$ ).

iris, tending to be enhanced where parasympathetic innervation is reduced. The reduction of light reflex amplitude and enhancement of response to pilocarpine $0.125 \%$ drops in the APACG group when compared to the DAC controls suggests relative parasympathetic dysfunction of the iris in APACG.

In the dark, the degree to which a pupil dilates can be regarded as predominantly a measure of sympathetic function. ${ }^{20}$ The reduced resting darkness pupil diameter in APACG eyes when compared to DAC controls indicates a degree of sympathetic dysfunction in addition to the parasympathetic dysfunction. Evidence for sympathetic dysfunction is relatively weak in this study as other measurements looking at predominantly sympathetic function ( $\mathrm{t} 3 / 4$, phenylephrine $2 \%$ testing) did not show the same difference between groups. The finding of reduced parasympathetic, and possibly sympathetic, dysfunction in APACG eyes is certainly consistent with the previously observed prolongation of pupil cycle time ${ }^{21}$ in acute angle closure patients. Pupil cycle time may be regarded as a rather imprecise measure of iris autonomic function ${ }^{22}$ which may be prolonged by either parasympathetic ${ }^{23}$ or sympathetic ${ }^{24}$ deficits, without allowing differentiation between the two. Reduction in both parasympathetic and sympathetic systems would have a marked effect on pupil cycle time

The significant correlation between ACD and some of the pupil tests requires consideration. ACD is known to decline with age ${ }^{1}$ and it is possible that the effects of ACD in this study are really a reflection of reduced autonomic responses occurring with age. The trend toward reduced autonomic responses with age has previously been demonstrated both for systemic autonomic function ${ }^{25}$ and pupil function. ${ }^{26,27}$ However, the results presented here show no significant correlation between age and ACD and, in addition, ACD correlates significantly with light reflex amplitude, resting darkness pupil diameter and pilocarpine response whereas age correlates with only pilocarpine response. These factors suggest that age and ACD have, to some extent, separate functions. It is unlikely that reduced ACD is the result of reduced autonomic function, particularly as decreased parasympthetic activity would tend to deepen the anterior chamber. ${ }^{28}$ Similarly, it is difficult to explain why reduced ACD should be associated with reduced autonomic function if not the result of it.

Table III. Difference between observed $(O)$ and expected $(E)$ values for pupil tests (mean $\pm S E M)$

\begin{tabular}{lccc}
\hline & APACG & SAC Control & DAC Control \\
\hline $\begin{array}{l}\text { O-E Resting darkness pupil diameter (mm, } \\
\text { corrected for ACD) }\end{array}$ & $-0.15 \pm 0.27$ & $+0.31 \pm 0.36$ & $-0.05 \pm 0.15$ \\
(NS) & $(\mathrm{NS})$ & $+0.19 \pm 0.07$ \\
$\begin{array}{l}\text { O-E Light reflex amplitude (mm, corrected } \\
\text { for diameter at foot of reflex) }\end{array}$ & $-0.16 \pm 0.09$ & $-0.12 \pm 0.13$ & $(\mathrm{p}<0.0005)$ \\
$\begin{array}{l}\text { O-E Light reflex amplitude (mm, corrected } \\
\text { for ACD) }\end{array}$ & $-0.04 \pm 0.10$ & $-0.016 \pm 0.13$ & $+0.031 \pm 0.08$ \\
O-E Pilocarpine 0.125\% miosis (mm, & $+0.25 \pm 0.14$ & $+0.39 \pm 0.37$ & $-0.35 \pm 0.27$ \\
$\quad$ corrected for age) & & $(\mathrm{NS})$ & $(\mathrm{p}<0.05)$ \\
$\begin{array}{l}\text { O-E Pilocarpine 0.125\% miosis (mm, } \\
\text { corrected for ACD) }\end{array}$ & $-0.33 \pm 0.33$ & $+0.09 \pm 0.39$ & $-0.031 \pm 0.22$ \\
\hline
\end{tabular}

Brackets indicate no significant difference (NS) or p value for unpaired t test between APACG and SAC control (shown in SAC control column) or APACG and DAC control (shown in DAC control column). 


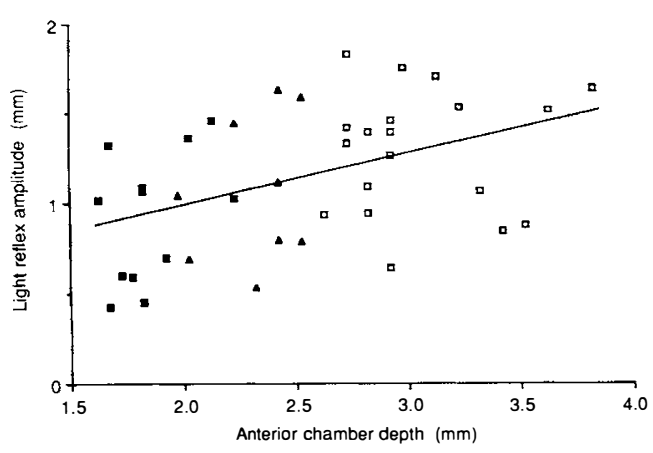

Fig. 2. Light reflex amplitude for $A P A C G(\mathbf{\square}), S A C$ controls ( $\mathbf{\Delta})$ and DAC controls $(\square)$ correlated signifcantly with anterior chamber depth $(r=0.40 p<0.05)$.

Increased mechanical apposition of the iris to the anterior lens surface might explain the reduced light reflex amplitude in eyes with shallow anterior chambers but this would tend to reduce rather than enhance the response to weak pilocarpine so a purely anatomical effect is unlikely. Perhaps the eye which has a physically different arrangement of anterior segment structures also has a different arrangement of autonomic innervation.

The correlation with ACD implies that iris autonomic function is reduced in eyes with shallow anterior chambers. This concept is supported by the lack of difference between APACG eyes (mean ACD $1.8 \mathrm{~mm}$ ) and SAC controls (mean ACD $2.3 \mathrm{~mm}$ ) for resting darkness pupil diameter, light reflex amplitude and pilocarpine miosis when the difference between APACG eyes and DAC controls (mean ACD $3.0 \mathrm{~mm}$ ) for the same parameters was significant. Reduced autonomic function

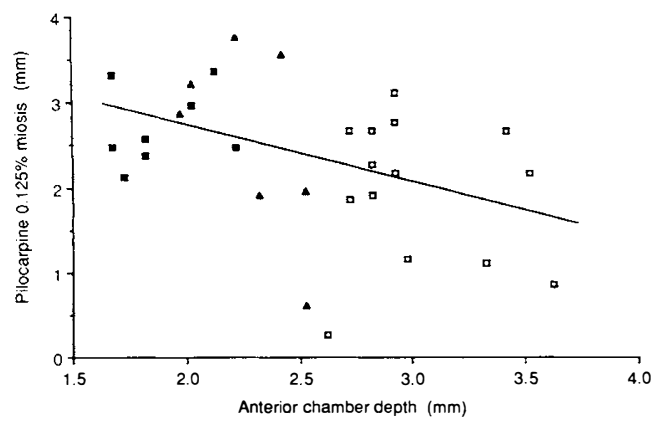

Fig. 3. Pilocarpine $0.125 \%$ miosis for APACG (ם), SAC controls $(\mathbf{\Delta})$ and DAC controls $(\square)$ showed a significant correlation with anterior chamber depth $(r=0.42 p<0.05)$. is therefore unlikely to be a specific finding in APACG but merely a reflection of the extremely shallow anterior chambers that such patients are known to have. ${ }^{1}$

The response to pilocarpine in SAC control eyes (Fig. 3) was widely variable between subjects. This indicates either innate variability of this test or the existence of two subsets of controls with shallow anterior chambers, namely, those with a supersensitive response to dilute pilocarpine and those without. A further study to assess whether the size of these responses has any predictive value for future acute angle closure would be of interest.

Because of the acute nature of primary angle closure episodes, pupil testing immediately after presentation may be difficult due to use of drugs and alteration of systemic autonomic function because of the stress of the illness. The finding of no significant difference between results of pupil studies performed in the same individuals immediately and some weeks after presentation indicates that neodymium-YAG laser iridotomy does not alter pupil responses to a significant degree and therefore, in future studies, it may be acceptable to recruit patients some time after treatment of the acute episode.

Glucose tolerance testing in this study did not produce evidence in support of a previous observation of decreased glucose tolerance in subjects with shallow anterior chambers and glaücoma. ${ }^{15}$ The difference between the studies may be explained by prospective exclusion of diabetics from the present study which would have reduced the incidence of glucose intolerance in the APACG subjects.

I am indebted to Professor S E Smith and Dr S A Smith for their advice and to the consultant ophthalmologists at St Thomas' Hospital who allowed me to study their patients. I thank Karen Clark and Claire Brooker for technical assistance and wish to gratefully acknowledge the support of the Iris Fund for Prevention of Blindness and the Special Trustees of St Thomas' Hospital.

\section{References}

${ }^{1}$ Duke-Elder S and Jay B: Diseases of the lens and vitreous, glaucoma and hypotony. In Duke-Elder $\mathrm{S}$ ed. System of Ophthalmology, London, Henry Kimpton 1969; Volume XI: 569-71. 
${ }^{2}$ Barkan O: Glaucoma: classification, causes and surgical control. Am J Ophthalmol 1938; 21: 1099117.

${ }^{3}$ Duke-Elder S and Jay B: Diseases of the lens and vitreous, glaucoma and hypotony. In Duke-Elder $\mathrm{S}$ ed. System of Ophthalmology, London Henry Kimpton 1969; Volume XI: 573.

${ }^{4}$ Simmons RJ and Dallow RL: Primary angle closure glaucoma. In Duane TD ed. Clinical Ophthalmology. Hagerstown, Harper and Row 1978; chapter 5 .

${ }^{5}$ Lowe RF: Acute angle-closure glaucoma precipitated by miotic plus adrenalîne eye-drops. Med J Aust 1966; 2: 1037-9.

${ }^{6}$ Clark CV and Mapstone R: Autonomic neuropathy in closed angle glaucoma. Research and Clinical Forums 1985; 7: 45-50.

${ }^{7}$ Mapstone R: Autonomic effects on aqueous outflow. Research and Clinical Forums 1981; 3: 35-9.

${ }^{8}$ Törnquist R: Chamber depth in primary acute glaucoma. Br J Ophthalmol 1956; 40: 421-9.

${ }^{9}$ Fison PN, Garlick DJ, Smith SE: Assessment of unilateral afferent pupillary defects by pupillography. Br J Ophthalmol 1979; 63: 195-9.

${ }^{10}$ Smith SA, Smith SE, Lazare R: An increased effect of pilocarpine on the pupil by application of the drug in oil. Br J Ophthalmol 1978; 62: 314-7.

${ }^{11}$ Lowenstein $\mathrm{O}$ and Loewenfeld IE: Role of sympathetic and parasympathetic systems in reflex dilatation of the pupil. Arch Neurol Psychiatr 1950; 64: 313-40.

12 Bourgon P and Thompson HS: How to test for cholinergic supersensitivity of the iris sphincter. In Thompson HS, Daroff R et al., eds. Topics in Neuro-Ophthalmology. Baltimore: Williams and Watkins 1979: 115-9.

13 Armstrong JR, Daily RK, Dobson HL, Girard LJ: The incidence of glaucoma in diabetes mellitus. Am J Ophthalmol 1960; 50: 55-68.

${ }^{14}$ Nielsen NV: The prevalence of glaucoma and ocular hypertension in type 1 and 2 diabetes mellitus. Acta Ophthalmol 1983; 61: 662-72.
${ }^{15}$ Mapstone $\mathrm{R}$ and Clark CV: Prevalence of diabetes in glaucoma. $\mathrm{Br}$ Med $J$ 1985; 291: 93-5.

16 Smith SE, Smith SA, Brown PM, Fox C, Sönksen $\mathrm{PH}$ : Pupillary signs in diabetic autonomic neuropathy. Br Med J 1978; 2: 924-7.

${ }^{17}$ Smith SA and Smith SE: Reduced pupillary light reflexes in diabetic autonomic neuropathy. Diabetologia 1983; 24: 330-2.

${ }^{18}$ Diabetes Mellitus. Technical Report Series 727. Geneva: World Health Organisation 1985.

${ }^{19}$ Lowenstein O and Loewenfeld IE: Mutual role of sympathetic and parasympathetic in shaping the pupillary reflex to light. Arch Neurol Psychiatr 1950; 64: 341-77.

${ }^{20}$ Smith SA: Pupillary function in autonomic failure. In Bannister R ed. Autonomic Failure. Oxford University Press 1988: 401.

${ }^{21}$ Clark CV and Mapstone R: Pupil cycle time in primary closed-angle glaucoma. Can $J$ Ophthalmol 1986; 21: 88-91.

22 Thompson HS: The pupil cycle time. J Clin Neuroophthalmol 1987; 7: 38-9.

${ }^{23}$ Martyn CN and Ewing DJ: Pupil cycle time: a simple way of measuring an autonomic reflex. $J$ Neurol Neurosurg Psychiatr 1986; 49: 771-4.

${ }^{24}$ Blumen SC, Feiler-Ofry V, Korczyn AD: The pupil cycle time in Horner's syndrome. J Clin NeuroOphthalmol 1986; 6: 232-5.

${ }^{25}$ Smith SE and Smith SA: Heart rate variability in healthy subjects measured with a bedside computer-based technique. Clin Sci 1981; 61: 379-83.

${ }^{26}$ Bourne P, Smith SA, Smith SE: Dynamics of the light reflex and the influence of age on the human pupil measured by television pupillometry. $J$ Physiol 1979; 293: 58P

${ }^{27}$ Smith SA and Smith SE: Evidence for a neuropathic aetiology in the small pupil of diabetes mellitus. Br J Ophthalmol 1981; 67: 89-93.

${ }^{28}$ Duke-Elder S and Abrams D: Ophthalmic optics and refraction. In Duke-Elder S ed. System of Ophthalmology. London: Henry Kimpton 1970; Volume V: 161-2. 